\title{
Glutamatergic Dysfunction of Lateral Habenula Promotes Depression
}

\author{
Bing Hu, Russell Fitzgerald ${ }^{\dagger}$
}

\begin{abstract}
Depression is one of the most prevalent neuropsychiatric disorders which can impair working capability of patients. However, the mechanism underlying the onset of depression remains to be elucidated. Traditionally, people pay much attention to monoamine system, but it was inadequate to understand pathophysiology of depression. Located in the dorsal dienchephalic conduction system, the habenula widely connects to brain regions associated with depression and has great impact on them. Accumulating evidence displays that lateral habenula (LHb) was hyperactive during depression in both clinic and preclinic studies. As a predominant excitatory neurotransmitter, glutamate distributes extensively in habenula, especially LHb. Aversive state can activate habenula, which in turn inhibits monoamine neurotransmitter release and induce depression. Therefore, glutamatergic excitatory neurotransmission in habenula plays a key role in depression etiology. Recent studies revealed that dysfunction of the process of glutamatergic neurotransmission triggered depression. Here, we report the progress of studies and recent findings that glutamatergic dysregulation in $\mathrm{LHb}$ contributes to depression, including dysregulation of glutamate level and glutamate transport, GABA/ glutamate ratio and $\mathrm{GABA}_{A}$ receptor, and AMPAR, CaMKIl as well as NMDAR. This will help us to understand the important role of glutamate in onset of depression and provides knowledge to guide future studies.
\end{abstract}

\section{Keywords}

Depression, Glutamate, lateral habenula, GLT-1, GABA/glutamate ratio, GABA, CaMKII, AMPAR

\section{Introduction}

The habenula is located above the thalamus and consists of the epithalamus, and it is divided into the lateral habenula (LHb) and the medial habenula (MHb) [1]. The habenula is an important relay station as it has extensive connections with the forebrain and the posterior brain. It receives afferents from the limbic system related to emotion and widely projects to multiple monoaminergic nuclei, which are involved in depression [2]. It has been reported the habenula is activated when the brain in the negative status and previous studies revealed that $\mathrm{LHb}$ is hyperactive in depression [3-6] and suppression of LHb by using deep brain stimulation ameliorated depressive symptoms in depressed patients $[5,7,8]$. Thus, the habenula has a well-documented and close relationship with neuropsychiatric depression (Figure 1).

Glutamate is an important excitatory neurotransmitter, and it is supposed to play an important role in the pathological process of many diseases, such as depression [9-12]. Glutamate is the predominant neurotransmitter in habenula as it is widely distributed within it. It is reported that almost all neurons in LHb are glutamatergic and many parts of $\mathrm{MHb}$ are glutamatergic as well [10]. Therefore, homeostasis of glutamate 


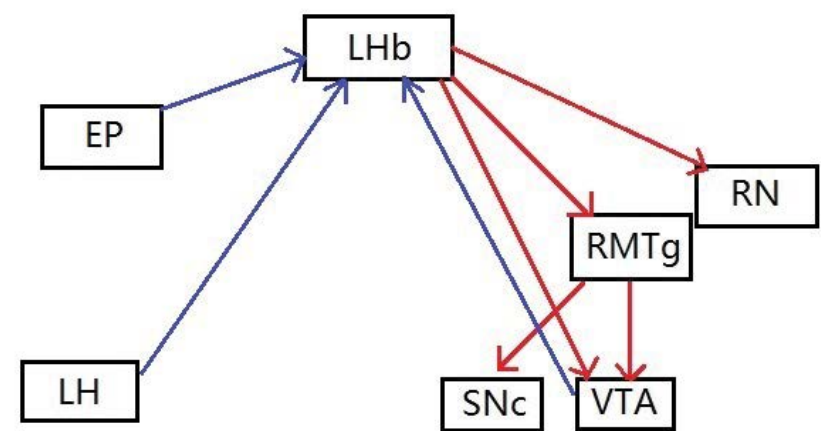

Figure 1: TAfferent and efferent glutamatergic connections of the lateral habenula (LHb).

The LHb receives inputs from the entopeduncular nucleus (EP), lateral hypothalamus (LH) and ventral tegmental area (VTA). The LHb sends outputs to the raphe nuclei (RN) as well as VTA. Moreover, the LHb projects to rostromedial tegmental nucleus (RMTg), which projects to VTA and substantia nigra pars compacta $(\mathrm{SNc})$, respectively. Purple lines indicate the afferent fibers to the LHb and red lines indicate the efferent connections from the $\mathrm{LHb}$.

is crucial for maintaining normal function of habenula, and if there is a dysregulation of glutamate neurotransmission, it will cause dysfunction of the habenula. It has been demonstrated that dysfunction of glutamate homeostasis in habenula existed in depression model [11]. However, it has not received much attention yet. This article reports recent discoveries and the progress of study on the dysregulation of glutamate neurotransmission in habenula in the onset of depression.

\section{Discussion}

\section{- Abnormality of glutamate level and glutamate transport}

The maintenance of physiological glutamate level in synaptic cleft depends on the balance of glutamate release and clearance [12]. Abnormality of any step in this process will cause dysfunction of glutamate. It has been found that extracellular glutamate level in LHb is substantially elevated in depressed rats induced by chronic mild stress, which in turn reduced 5-HT level in dorsal raphe nucleus [11]. Whether this enhancement of glutamate level in LHb is due to over release of glutmatergic neuron in LHb or dysregulation of glutamate clearance, remains to be clarified in further study.

After glutamate is released into the synaptic cleft, the glial glutamate transporter GLT-1 of the astrocyte rapidly uptakes the glutamate to minimize overflow [13]. Therefore, GLT1 is essential for maintaining normal level of glutamate. Since almost all neurons in the LHb are glutamatergic and the LHb plays a key role in pathophysiology of depression, alteration of GLT-1 in the LHb will contribute to depression.
There is evidence that the downregulation of astrocytic GLT-1 results in LHb hyperactivity [13] and knockout of LHb GLT-1 leads to depressive behaviors [14]. A recent study further investigated the role of maladaptation of GLT1 in a depressive state induced by alcohol withdrawal and elucidated that GLT-1 in the $\mathrm{LHb}$ decreased. This reduction was restored by administering ceftriaxone, which in turn normalized the hyperexcitability of LHb and ameliorated depressive behavior [14].

This indicates GLT-1 could be a promising target to treat psychiatric comorbidity of ethanol withdrawal. Additionally, it will be interesting to know for the future study if there are also other excitatory amino acid transporters involved in the hyperactivity of the LHb in depression.

\section{- Dysfunction of GABA/glutamate ratio and $\mathrm{GABA}_{\mathrm{A}}$ receptor in $\mathrm{LHb}$}

GABA is the major inhibitory neurotransmitter in the brain, which decreases neuron activity and avoids neuron overfiring [15]. As the level of GABA and glutamate is important to balance inhibition and excitation of $\mathrm{LHb}$, once the GABA/glutamate ratio loses its balance, depression can occur. Furthermore, as $\mathrm{GABA}_{\mathrm{A}}$ receptor mediates fast inhibitory response of GABA in LHb, its abnormality also initiates depression [16].

Investigators discovered synaptic terminals projecting from entopeduncular nucleus (EP) to $\mathrm{LHb}$ can co-release GABA and glutamate and co-existence even be detected from certain individual vesicles [17]. The GABA/glutamate ratio controls the excitability of LHb. It was found that GABA of co-release remarkably reduced in animal models of human depression and GABA/ 
glutamate ratio decreased, which enhanced excitatory transmission and activity of $\mathrm{LHb}$ neurons. Conversely, after depressive animals were treated with antidepressant chronically, the GABA/glutamate ratio recovered and depressive behavior alleviated [17-19]. This proved the dysregulation of the GABA/glutamate ratio in the LHb caused hyperactivity of the LHb, which in turn induced depression. This suggests that drugs which can balance GABA function could improve depression.

\section{- Dysregulation of AMPAR, CaMKII and NMDAR in LHb}

Since $\mathrm{LHb}$ neurons are almost all glutamatergic [20], GluA1-containing a-amino-3-hydroxy5-methyl-4-isoxazolepropionic acid receptors (AMPAR), a subtype of ionotropic glutamate receptors, is largely expressed in glutamatergic synapses in $\mathrm{LHb}$, which can be regulated by $\mathrm{Ca}^{2+} /$ calmodulin-dependent protein II (CaMKII) [21]. AMPAR is composed of four subunits (GluA1-4) and the GluA1 subunit is expressed mostly in the LHb [22]. $\mathrm{Ca}^{2+} /$ calmodulin-dependent protein II (CaMKII) is a $\mathrm{Ca}^{2+}$-activated enzyme and its activation heightens glutamatergic synaptic transmission by the phosphorylation of the Ser831 residue of the GluA1 subunit of AMPAR [23] and by promoting AMPAR insertion into synapse [24].

Previous research has identified that the level of $\beta \mathrm{CaMKII}$ in the LHb is enhanced in depressive animal models of learned helpless and chronic mild stress and even artificial overexpression of $\beta \mathrm{CaMKII}$ in the LHb in unstressed animals also induced depressive symptoms by increasing synaptic transmission and electrophysiology activity of LHb neurons, which can be downregulated by antidepressants [6]. This could be due to the reason that elevated $\beta$ CaMKII strengthens AMPAR synaptic transmission and over activates LHb. In addition, although $\beta$ CaMKII has properties of both kinase and a structural scaffolding protein at the synapses [25], the kinase function of $\beta$ CaMKII was required for inducing depression [6].

A recent study identified enhancement of AMPAR and CaMKII activity in LHb in alcohol withdrawal animals from chronic intermittent ethanol consumption with comorbidity of depression [21]. Suppression of AMPAR and
CaMKII activity in the LHb improved depressive behavior and alcohol drinking and seeking behaviors. In contrast, activating AMPAR in the $\mathrm{LHb}$ triggered depressive behavior even in alcohol naïve animals. These outcomes revealed CaMKII-AMPAR signaling pathway is essential to mitigate the depressive-like behavior concomitant with alcohol withdrawal. Moreover, the very latest study revealed that the N-methyl-d-aspartate receptor (NMDAR) plays an essential role in the onset of depressive disorder [26].

This study demonstrated that overactivation of NMDAR changed the firing pattern of LHb neuron to intensive burst firing, which enhanced LHb activity and initiated depressive behavior [26].

These studies indicate the potential of AMPAR, CaMKII and NMDAR as therapeutic targets, and guide future drug development.

\section{Conclusion}

The present article reports previous study and current progress of the dysregulation of glutamate neurotransmission pathway in LHb which contribute depression etiology. Taken together, dysregulation in any step of glutamate transmission will lead to depression. It was elucidated downregulation of astrocytic GLT-1 caused accumulation of glutamate and depression. The reduction of GABA/glutamate ratio and the abnormality of $\mathrm{GABA}_{\mathrm{A}}$ receptor induced depression as well. Furthermore, upregulation of CaMKII also triggers depression by strengthening AMPAR function.

These findings enrich our knowledge of mechanism of depression. However, there are still limitations for current studies. For example, what mechanism induces over release of glutamate, whether other excitatory amino acid transporters participate in the hyperactivity of the LHb and which NMDA receptor subtypes in $\mathrm{LHb}$ involve in depressive disorder, which remain to be clarified in future studies. These suggest that additional study in other glutamateassociated mechanisms may be helpful for further understanding depression. Moreover, exploring the approach targeting the glutamate neurotransmission process may be a promising treatment for depression. 


\section{References}

1. Hikosaka O. The habenula: From stress evasion to value based decision-making. Nat. Rev. Neurosci 11(7), 503-513 (2010).

2. Lecca S, Meye FJ, Mameli M. The lateral habenula in addiction and depression: An anatomical, synaptic and behavioral overview. Eur. J. Neurosci 39(7), 1170-1178 (2014).

3. Morris JS, Smith KA, Cowen PJ, et al. Covariation of activity in habenula and dorsal raphe nuclei following tryptophan depletion. Neuroimage 10(2), 163-172 (1999).

4. Shumake J, Edwards E, Gonzalez-Lima F. Opposite metabolic changes in the habenula and ventral tegmental area of a genetic model of helpless behavior. Brain. Res 963(1-2), 274-281 (2003).

5. Li B, Piriz J, Mirrione M, Chung C, et al. Synaptic potentiation onto habenula neurons in the learned helplessness model of depression. Nature 470(7335), 535-539 (2011).

6. Li K, Zhou T, Liao L, et al. beta CaMKII in lateral habenula mediates core symptoms of depression. Science 341(6149), 1016-1020 (2013).

7. Sartorius A, Kiening KL, Kirsch P, et al. Remission of major depression under deep brain stimulation of the lateral habenula in a therapy-refractory patient. Biol. Psychiatry 67(2), e9-e11 (2010).

8. Meng $\mathrm{H}$, Wang $\mathrm{Y}$, Huang $\mathrm{M}$, et al. Chronic deep brain stimulation of the lateral habenula nucleus in a rat model of depression. Brain. Res 1422, 32-38 (2011).

9. Shirayama Y, Takahashi M, Osone F, et al. Myo-inositol, glutamate and glutamine in the prefrontal cortex, hippocampus and amygdala in major depression. Biol.
Psychiatry. Cogn. Neurosci Neuroimaging 2(2), 196-204 (2017).

10. Zhang BB, Du JL. The structure and function of habenula. Acta. Physiologica. Sinica 69(5), 623-636 (2017).

11. Yang LM, Hu B, Xia YH, et al. Lateral habenula lesions improve the behavioral response in depressed rats via increasing the serotonin level in dorsal raphe nucleus. Behav. Brain. Res 188(1), 84-90 (2008).

12. Gegelashvili G, Robinson MB, Trotti D, et al. Regulation of glutamate transporters in health and disease. Prog. Brain. Res 132(1), 267-286 (2001).

13. Kang S, Li J, Bekker A, et al. Rescue of glutamate transport in the lateral habenula alleviates depression-and anxiety-like behaviors in ethanol-withdrawn rats. Neuropharmacology 129(1), 47-56 (2018).

14. Cui W, Mizukami H, Yanagisawa $M$, et al. Glial dysfunction in the mouse habenula causes depressive-like behaviors and sleep disturbance. J. Neurosci 34(49), 1627316285 (2014).

15. Isaacson JS, Scanziani M. How inhibition shapes cortical activity. Neuron 72(2), 231243 (2011).

16. Wang T, Zhang L, Zhang QJ, et al. Involvement of lateral habenula a1 subunit-containing GABAA receptormediated inhibitory transmission in the regulation of depression-related behaviors in experimental Parkinson's disease. Neuropharmacology 116(1), 399-411 (2017).

17. Shabel SJ, Proulx CD, Piriz J, et al. GABA/ glutamate co-release controls habenula output and is modified by antidepressant treatment. Science 345(6203), 1494-1498 (2014).

18. Freo U, Ori C, Dam M, et al. Effects of acute and chronic treatment with fluoxetine on regional glucose cerebral metabolism in rats: implications for clinical therapies. Brain. Res 854(1-2), 35-41 (2000).

19. Jarosik J, Legutko B, Unsicker K, et al. Antidepressant-mediated reversal of abnormal behavior and neurodegeneration in mice following olfactory bulbectomy. Exp. Neurol 204(1), 20-28 (2007).

20. Aizawa H, Kobayashi M, Tanaka S, et al. Molecular characterization of the subnuclei in rat habenula. J. Comp. Neurol 520(18), 4051-4066 (2012).

21. Jing Li, Seungwoo Kang, Rao Fu, et al. Inhibition of AMPA receptor and CaMKII activity in the lateral habenula reduces depressive-like behavior and alcohol intake in rats. Neuropharmacology 126(1), 108-120 (2017).

22. Meye FJ, Lecca S, Valentinova K, et al. Synaptic and cellular profile of neurons in the lateral habenula. Front. Hum. Neurosci 16(7), 860 (2013)

23. Barria A, Muller D, Derkach V, et al. Regulatory phosphorylation of AMPA-type glutamate receptors by CaM-KII during long-term potentiation. Science 276(5321) 2042-2045 (1997).

24. Hayashi Y, Shi SH, Esteban JA, et al. Driving AMPA receptors into synapses by LTP and CaMKII: requirement for GluR1 and PDZ domain interaction. Science 287 (5461), 2262-2267 (2000).

25. Okamoto K, Narayanan R, Lee $\mathrm{SH}$, et al. The role of CaMKII as an F-actinbundling protein crucial for maintenance of dendritic spine structure. Proc. Natl. Acad. Sci. U. S. A 104(15), 6418-6423 (2007).

26. Yang Y, Cui Y, Sang K, et al. Ketamine blocks bursting in the lateral habenula to rapidly relieve depression. Nature 554(7692), 317322 (2018) 